\title{
ACCURACY ASSESSMENT OF FINITE-VOLUME AND HIGH-ORDER COMPACT METHODS FOR LES OF COMBUSTOR FLOW-FIELDS
}

\author{
VISHAL SAINI ${ }^{1}$, HAO XIA ${ }^{2}$ AND GARY J. PAGE ${ }^{3}$ \\ ${ }^{1}$ Loughborough University \\ Dept. of Aeronautical and Automotive Engineering, Loughborough University, LE11 3GR, UK \\ v.saini@lboro.ac.uk \\ ${ }^{2}$ Loughborough University \\ Dept. of Aeronautical and Automotive Engineering, Loughborough University, LE11 3GR, UK \\ h.xia@lboro.ac.uk \\ ${ }^{3}$ Loughborough University \\ Dept. of Aeronautical and Automotive Engineering, Loughborough University, LE11 3GR, UK \\ g.j.page@lboro.ac.uk
}

Key words: High-order, Spectral/hp, OpenFoam, Comparison, Turbine, Combustor, Large-Eddy, Simulation, Turbulence.

\begin{abstract}
The Large-Eddy Simulations (LES) play an important role in the study and design of gas turbine combustors. In order to bring the cost of LES down, compact high-order accurate solvers that can handle complex geometry are a promising method. However, their accuracy vs cost benefit as compared to the standard finite-volume solvers is unclear when it comes to under-resolved hybrid unstructured meshes. These meshes are required to represent the industrial combustor geometries. In this work, a high-order spectral/hp (Nektar++) solver is compared to a standard finite-volume (OpenFoam) solver for a fixed cost. Two combustor-relevant configurations are employed, port-flow and swirling flow. It is found that high-order solver provides moderate accuracy improvements in terms of the mean results and significant improvements for the unsteady results.
\end{abstract}

\section{INTRODUCTION}

Tightening emission regulations is one of the key drivers of the combustor technology for civil aircraft gas turbine (GT) engines. The flow inside a GT combustor is kept highly turbulent to achieve efficient combustion [1]. Main flow features include strong swirl and jets-in-cross-flow, resulting in high unsteadiness levels. For the understanding and design process of the combustor flows, numerical simulations play an important role. The presence of unsteadiness limits the applicability of standard relatively cheaper Reynolds Averaged Navier-Stokes (RANS) or unsteady-RANS simulations [1]. On the contrary, the Direct Numerical Simulations (DNS) are prohibitively expensive for realistic flow conditions. The Large-Eddy Simulations (LES) are an intermediate option that is becoming more accessible due to rapidly growing cheap computing.

One of the challenges for industrial LES is to handle complex combustor geometries while providing 
required flow-solution accuracy. Currently, the industrial LES is performed using second-order (or 'loworder') accurate finite-volume (FV) packages that can use hybrid unstructured grids to represent complex geometries. However, due to relatively large numerical errors, solvers based on second-order schemes may not be able to provide required accuracy for a given cost, or can be expensive for a given accuracy. The high-order accurate (order $\geq 3$ ) schemes are a promising way to provide required accuracy/cost benefits [2]. Thus, any required evaluations and improvements are worth investing in. The elementbased compact schemes such as spectral-element (SE), spectral/hp and flux-reconstruction (FR) are of special interest as they combine high-order accuracy with geometrical flexibility.

A systematic effort to study the accuracy vs cost benefit of the high-order schemes is undertaken by highorder CFD workshops [2]. However, conclusive results are found solely on relatively simple unsteady flow configurations. Recently, Capuano et al. [3] compared the performance of a high-order spectralelement solver Nek5000 and a second-order OpenFoam solver for the DNS of oscillatory plane jet flow. It is reported that while tenth-order accurate $N e k 5000$ is 1.5 to 4 times slower for a given number of solution points, it obtains equivalent accuracy for 7 times lower number of solution points. This is favourable for Nek5000 but, the geometry is again simplistic and the meshes are highly resolving. Vermeire et al. [4] compare high-order FR package $P y F R$ to a commercial second-order solver on a slightly more complex configuration, i.e., SD7003 aerofoil. Fifth-order $P y F R$ obtains more accurate pressure coefficient albeit with six times higher cost. Thus, the benefit is not clear.

To the authors' knowledge, the existing conclusive comparative studies are limited to simple geometries and/or well-resolved LES scenarios, whereas the industry usually works with complex geometries and

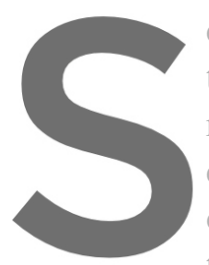
coarse meshes. It was the accuracy benefit avail relevant scenario. The compared to a standard order solver is based on to the OpenFoam [7] framework
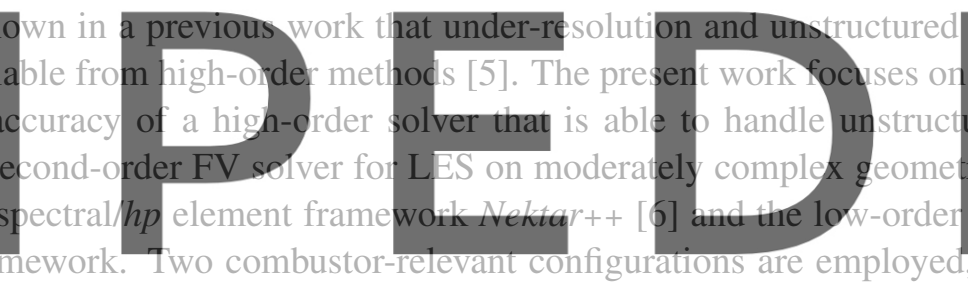

swirling flow. The im is to objectively evaluate the accuracy benefit of the high-order, accoter

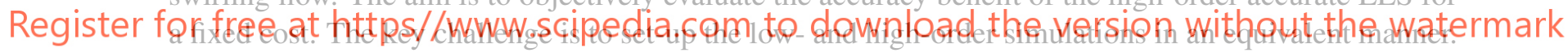

The set-up, in addition to the accuracy and cost definition, is described in Section 2. The test cases are detailed in Section 3. Finally, the results and conclusions are presented in Section 4 and 5 respectively.

\section{METHOD}

The details of the governing equations, solvers and cost/accuracy metrics are described in this section. The requirement is to evaluate the accuracy for a fixed cost using available open-source software packages. Two realistic and industrially relevant test cases, combustor port-flow and swirler, are employed for the comparison. Experimental data from multiple campaigns is available for both the cases. The first case consists of hybrid mesh (hexahedra, tetrahedra and pyramids) whereas the second one of hexahedra only. The latter is to provide a baseline for the future hybrid mesh simulations. The LES are run for each test case using the finite-volume and spectral $/ h p$ solvers incurring a nearly identical cost (difference of less than 4\%). The simulations are set up from an industrial perspective i.e. the meshes are underresolved, and the simulation parameters such as solver schemes, sub-grid scale models are kept close to the standard industrial practices. 
The second-order FV solver comes from the OpenFoam [7] framework and the high-order spectral $/ h p$ solver from Nektar++ [6]. The mentioned solvers are apt for the present study because of their ability to handle mixed type of grid elements necessary for complex geometries. Fourth polynomial order (or fifth order accuracy) is used within Nektar++. The polynomial basis is the modified Jacobi basis [6]. The flow is modelled using the unsteady incompressible Navier-Stokes equations. The effect of the unresolved sub-grid scales (SGS) is modeled using an eddy-viscosity model within the OpenFoam solver (explicit LES approach) and, an artificial spectral viscosity approach in the Nektar ++ solver. The former is a widely adopted choice within the currently used industrial CFD software, mainly due to its physical basis, simplicity and robustness. On the other hand, there is evidence that using artificial spectral viscosity instead of eddy-viscosity SGS model might be better suited for high-order schemes from the accuracy point of view [8]. Thus, the spectral viscosity approach is adopted for the spectral $/ h p$ solver. The details of the subgrid scale treatments are now discussed.

The eddy-viscosity $v_{t}$ is prescribed with the Wall-Adapting Local Eddy-viscosity (WALE) model [9] as

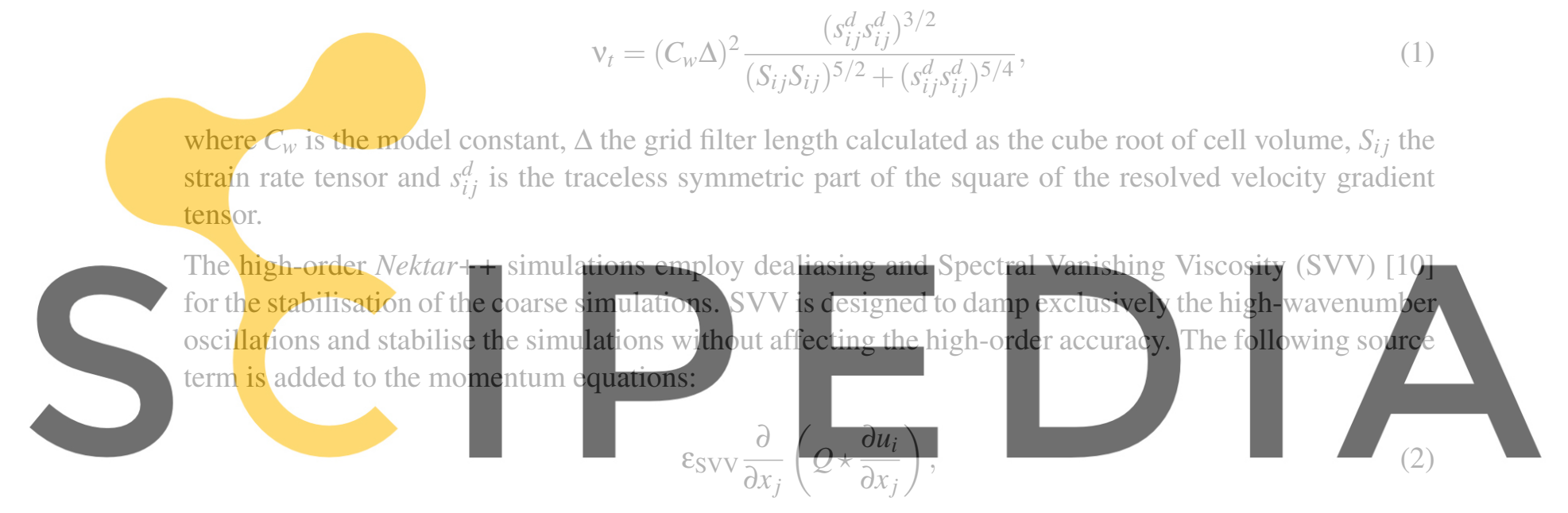

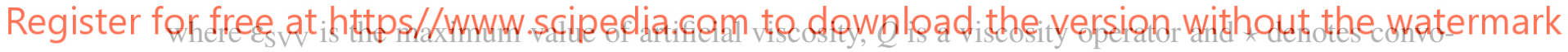

lution. For details in the spectral/hp context, see [10]. The use of SVV is crucial here because the high-

order solver uses the continuous-Galerkin approach, where unlike the discontinuous-Galerkin method, an inherent inter-elemental dissipation mechanism is not available. Several examples of successful applications of SVV for the LES of practical flows exist, e.g. [11, 12].

A recently formulated SVV operator [13] with a power-kernel is used. In this operator, the $\varepsilon_{S V V}$ magnitude is proportional to a measure of local advection speed $U_{l}$ and local mesh spacing $h / \mathrm{P}$, which gives

$$
\varepsilon_{\mathrm{SVV}}=\varepsilon_{0} U_{l} h / \mathrm{P}
$$

where $\varepsilon_{0}$ is a fixed parameter, $h$ is the local elemental mesh size and $\mathrm{P}$ is the polynomial order. The spectral variation $(\stackrel{*}{)}$ ) of the kernel is defined as:

$$
\hat{Q}(m)=(m / \mathrm{P})^{\mathrm{P}_{\mathrm{SVv}}}, 0 \leq m \leq \mathrm{P},
$$

where $m$ is the mode index and $\mathrm{P}_{\mathrm{SVV}}$ relates to the activation threshold. As in the original paper [13], $\mathrm{P}_{\mathrm{SVV}}=\mathrm{P} / 2=2$ is used and $\varepsilon_{0}=3.0$ is found to provide a stable run. 
Table 1: Comparison of the low- and high-order solvers.

\begin{tabular}{llllll}
\hline Framework & Space Discretization & Order & Solver & Pres.-Vel. Algorithm & SGS model \\
\hline OpenFoam & Finite-Volume & 2 & pimpleFoam & PIMPLE/PISO & WALE [9] \\
Nektar ++ & Spectral/hp & 5 & IncNavierStokesSolver & Velocity Correction & SVV [10] \\
\hline
\end{tabular}

A broad comparison of the two solvers is summarised in Table 1. The pressure-velocity algorithms are chosen to obtain the highest performance with stability from each solver. The PIMPLE algorithm with two SIMPLE outer correctors is found suitable for the port flow case whereas a single outer corrector (i.e. PISO) is sufficient for the swirler case. The time schemes used are second-order accurate backward schemes. In the OpenFoam solver it is a conditionally-stable second-order implicit scheme, and in the Nektar++ solver it is a stiffly-stable second-order implicit-explicit scheme. The second-order accuracy and small time-steps used in this study ensure that time discretization error is small as compared to that of spatial discretization.

The quantities that are kept similar for the two solvers are: flow definition, computational domain, spatial mesh distribution, time-step size and linear solver residual tolerances $\left(1 \times 10^{-7}\right.$ for velocity and $1 \times 10^{-6}$ for pressure). For pressure iterations, OpenFoam uses GAMG and Nektar++ uses low-energy block preconditioner for the conjugate gradient solver.

The definition of the cost in the present study is simply the nominal CPU wall clock time for a simulation to finish. This is consid nodes with 28 cores in connected with Mellanox in terms of the error generated to the available experin combustion processes.
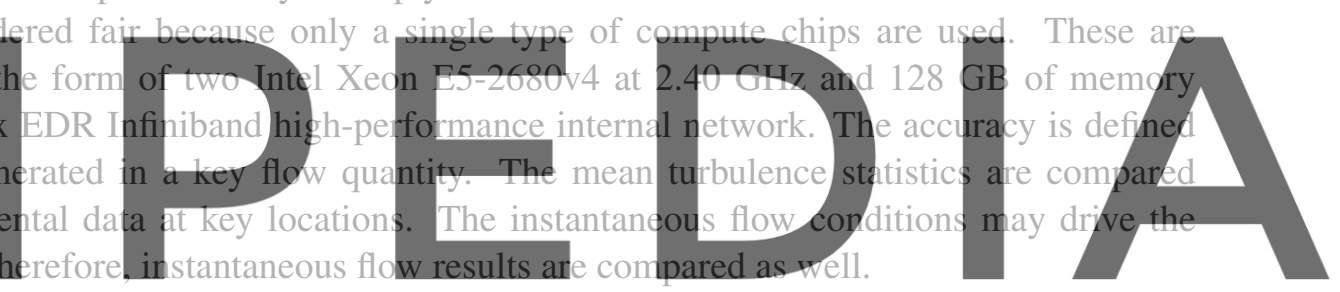

Register för free afAtESS//www.scipedia.com to download the version without the watermark

Two test cases are considered, port-flow and swirler. Their relevance to combustors and their specifications are described in the following. Note that from hereon, the OpenFoam and Nektart+ solvers are denoted by $O F$ and $N p p$ respectively.

\subsection{Port flow}

Dilution jets in crossflow direction are employed in rich-burn type gas turbine combustors. They provide the oxidant, support the recirculation zone (RCZ) and enhance the turbulent mixing. This improves the overall combustion efficiency [1]. A representative flow configuration has been experimentally studied in the past using laser techniques $[14,15]$. The particle image velocimetry (PIV) measurements from Hollis [15] are used as a reference here. The experimental test-section test section consisted of two concentric pipes where the inner core pipe is fed from the outer annulus through six equally spaced circular ports. This results in six jets impinging onto each other. A schematic of a cross-section is shown in Figure 1.

The flow condition is defined by three parameters, (i) mass flow rate bleed ratio, i.e. $B=\dot{m}_{p} / \dot{m}_{a}$; (ii) bulk velocity ratio of the port-jet and the core inlet, i.e. $\alpha=U_{p} / U_{c}$ and; (iii) Reynolds number of the port-jet, 


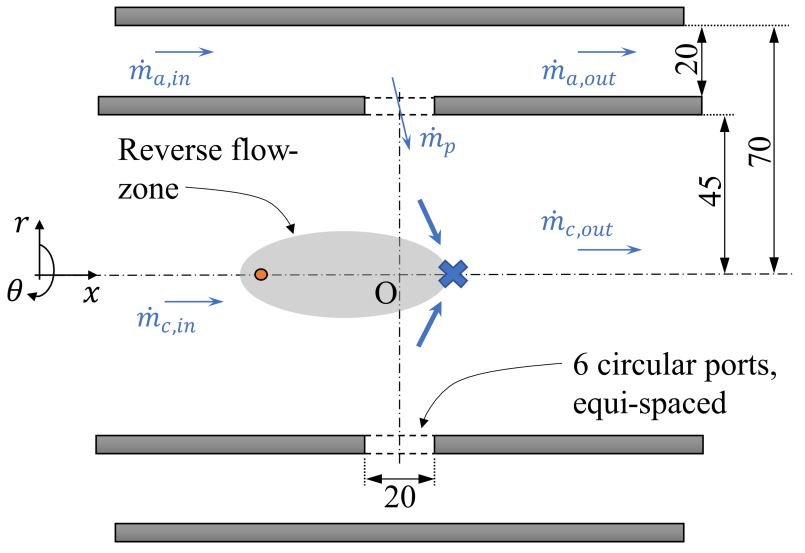

$$
\begin{aligned}
& \text { O : origin } \\
& \downarrow \text { : Port jets } \\
& \checkmark: \text { Jets' impinging point } \\
& \circ: \text { Point probe }
\end{aligned}
$$

All dimensions in $\mathrm{mm}$.

Figure 1: Schematic and flow features of the combustor port flow case.

i.e. $R e_{p}=U_{p} D_{p} / v$, where $D_{p}$ is the diameter of the port. The subscripts $a, c$ and $p$ refer to annulus, core and ports respectively. For the present case, $B=0.5, \alpha=5$ and $R e_{p} \approx 2.25 \times 10^{4}$ with $U_{c}=0.2 \mathrm{~m} / \mathrm{s}$. The parameters values are set such that the momentum ratio of the port-jets to the axial bulk cross-flow is representative of practical combustors (section 4.1 in [1]). The working fluid in the experimental rig as well as the present simulations is water with kinematic viscosity $\mathrm{v}=8.9 \times 10^{-7} \mathrm{~m}^{2} / \mathrm{s}$.

The main flow features include the reverse-flow or recirculation zone (RCZ) in the core, high turbu-

The LES domain extend

the axial direction [5].

cost and the spatial distribu
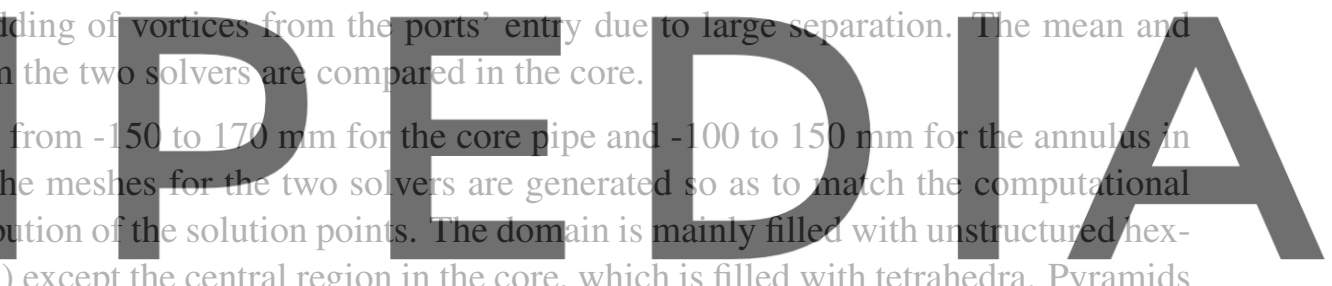

Register naturally appear for the transition. Figure 6 compares the cross-section of the volume meshes The total Register for free at https/JWWw.scipedia. Com to download the version without the watermark elements which amounts to approximately 8.4M solution points.

The velocity profiles available from the reference experiment [15] are imposed at the inlets. The inlet turbulent fluctuations are not modelled since the turbulence generation is dominated by the jets' impingement and shear layers. At the annulus outlet, a scaled inlet velocity profile is imposed to obtain target mass split i.e. $B=0.5$. Finally, the core exit is a fixed pressure outlet and the walls are no-slip boundaries. The $O F$ is run with blended (40\% upwind and $60 \%$ central) scheme for the advection term as the simulation is unstable with higher central contribution for this complex case. The time-step is $5.0 \times 10^{-6}$ $\mathrm{s}$ for both $O F$ and Npp.

The flow-through time based on the core diameter and bulk inlet velocity is $T_{F}=0.09 / U_{c}=0.45 \mathrm{~s}$. Note that a residence time of $0.363 \mathrm{~s}$ is reported by Spencer \& Adumitroaie [16] for the same configuration. In the present study, the flow is first developed for several flow-through time units i.e. 5.5TF. Finally, the statistics are collected over the time span of $6.7 T_{F}$ i.e. $3 \mathrm{~s}$. This sampling time is higher as compared to the previous LES studies on this configuration ( $2 \mathrm{~s}$ in [16] and $1.51 \mathrm{~s}$ in [17]). The simulations are run on 336 cores (12 nodes). The approximate total memory usage is around 500 GB for Npp and $200 \mathrm{~GB}$ 


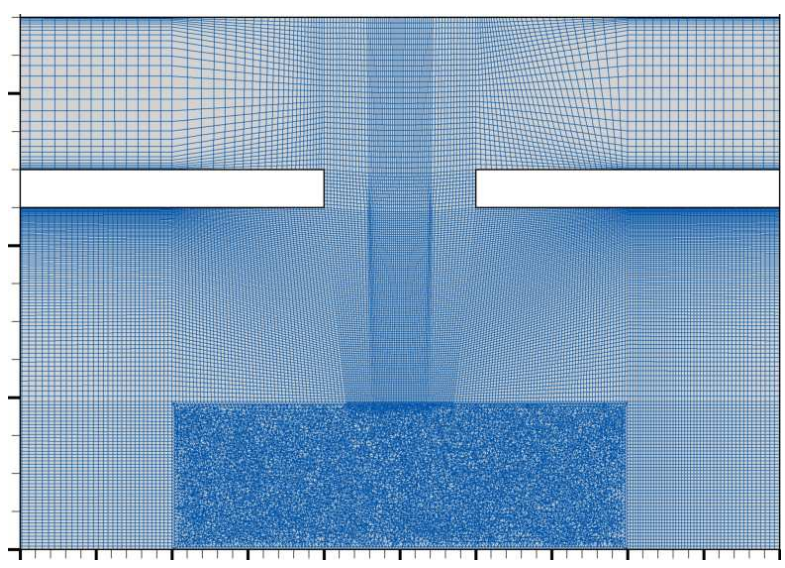

(a) $16 \mathrm{M}$ cell mesh $O F$

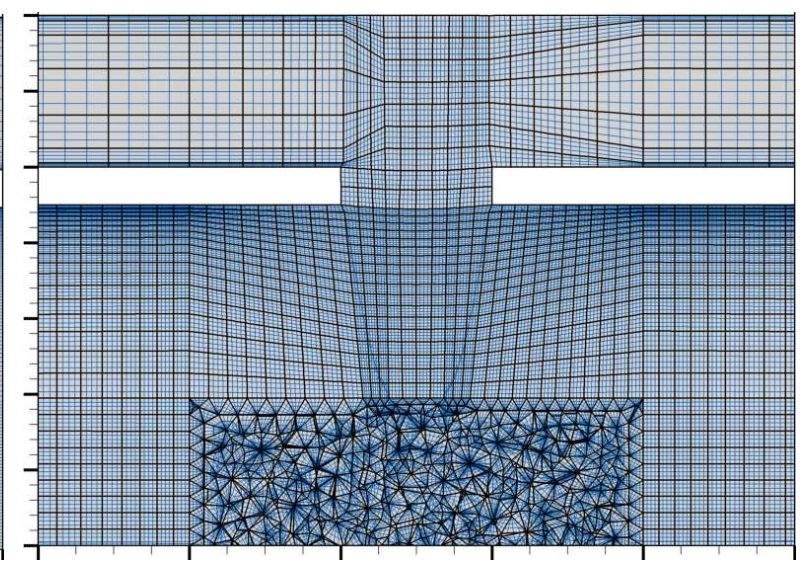

(b) $8.5 \mathrm{M}$ solution points $\mathrm{Npp}$

Figure 2: Comparison of the second-order OpenFoam and P4 Nektar++ mesh on a central cross-section of port flow case. Dark lines represent elements in (b). M denotes million.

for $16 \mathrm{M}-O F$. These memory requirements did not pose a problem as the total available memory on the 12 nodes is around $1450 \mathrm{~GB}$. In absolute terms, the computational cost of collecting statistics $\left(6.7 T_{F}\right.$ or 600,000 time-steps) is around 60,000 core hours.
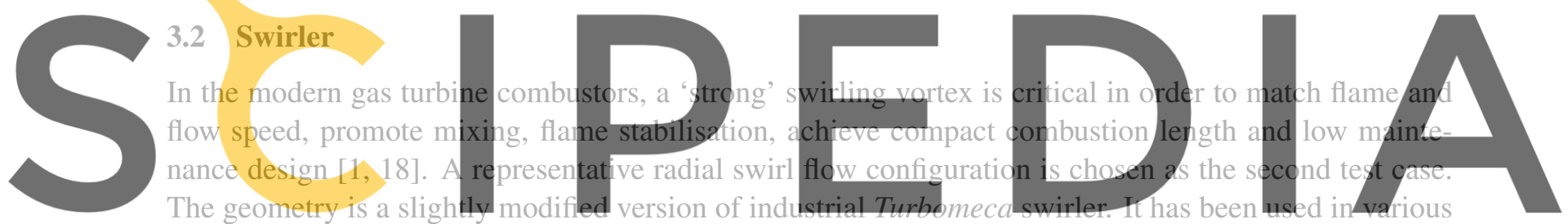

experimental and numerical studies in the past $[19,20,21]$. The isothermal PIV experiment of Cheng et

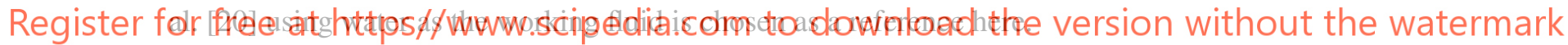

A schematic of the experimental section is shown in Figure 3 (for details, see [22]). There are two inlets, the outer one that feeds the swirler, and the centrai one feeding the centrai pipe. The filow from the outer (annular) inlet enters the radial swirlers (Figure 3b) where tangential component is added to the velocity. The working fluid then passes through the swirler passage $\left(-1<x / D_{s}<0\right)$ and is finally discharged into the suddenly expanded dump chamber $(x>0)$. The level of confinement provided by the chamber is close to that found in typical aero-engine combustors [22]. The flow from the central pipe issues as a jet in the dump chamber as well. The central jet is a representative fuel stream in case of non-premixed combustion [21]. A cylindrical constriction is present towards the exit in order to avoid overly elongated swirl effects [22].

The main flow features include strong swirl, the resulting central recirculation zone, flow separation at the inner surface of swirler passage and 'two-strong two-weak' precessing vortices (PVC) [23]. The flow condition is defined defined by three parameters: (i) Reynolds number of the central jet stream, $R e_{j}=U_{j} D_{j} / \mathrm{v}$, where $U_{j}$ is the bulk jet velocity and $D_{j}$ the jet diameter; (ii) Reynolds number of the swirl stream, $R e_{s}=U_{s} D_{s} / \mathrm{v}$, where $U_{s}$ is the bulk swirler velocity and $D_{s}$ the outer diameter and; (iii) 


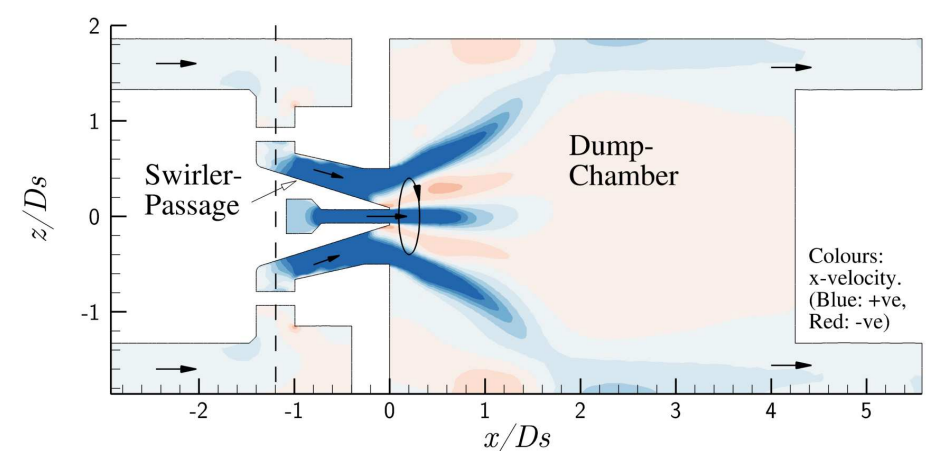

(a) axial-radial

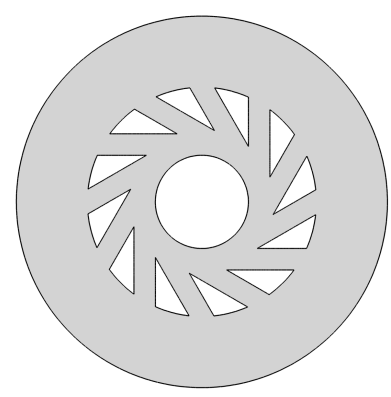

(b) swirlers in radial-azimuthal

Figure 3: Experimental test section for swirler case. The outer diameter of the swirler passage at $x=0$ is denoted by $D_{S}$ and is equal to $37.63 \mathrm{~mm}[19]$.

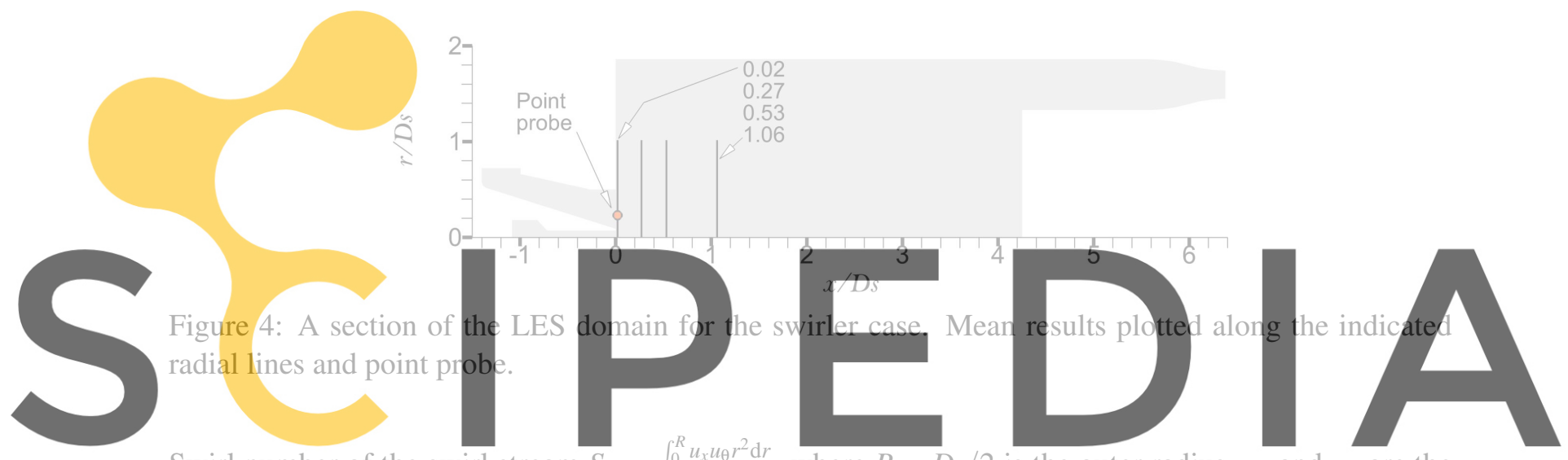

Swirl number of the swirl stream $S n=\frac{\int_{0}^{R} u_{x} u_{\theta} r^{2} \mathrm{~d} r}{R \int_{0}^{R} u_{r}^{2} \mathrm{~d} r}$, where $R=D_{S} / 2$ is the outer radius, $u_{x}$ and $u_{\theta}$ are the

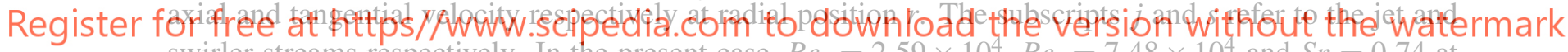
swirler streams respectively. In the present case, $R e_{j}=2.59 \times 10^{4}, R_{S}=7.48 \times 10^{4}$ and $S n=0.74$ at the swirler passage exit.

Figure 4 illustrates a section of the full three-dimensional domain used for the LES runs. Following previous studies [20,24], the radial vanes are not modelled explicitly to save computational costs. The effect of the vanes is instead included by imposing a velocity profile obtained from a precursor URANS simulation. It is verified that the precursor simulation provides acceptable match to the global quantities $R e_{j}, R e_{s}$ and $S n$. Further, a smooth non-uniform velocity profile similar to Ref. [12] is applied to the central jet inlet. A convergent section is added near the outlet so as to accelerate any corner separation vortices that may result in negative flow at the outlet [20,24]. Finally, the outlet is at a fixed pressure and the walls are no-slip boundaries.

The meshes for the two solvers are generated to match the cost and the spatial distribution of the solution points. The domain is filled with hexahedral cells (or elements). Recall that this is to establish a baseline for a future case with completely unstructured hybrid mesh. The $O F$ mesh is $5.7 \mathrm{M}$ cells whereas the $N p p$ mesh consists of 31,000 elements, i.e. approximately $2 \mathrm{M}$ solution points. Note that the total solution points ratio between $O F$ and $N p p$ is higher here (around 3) as compared to the port flow case where 


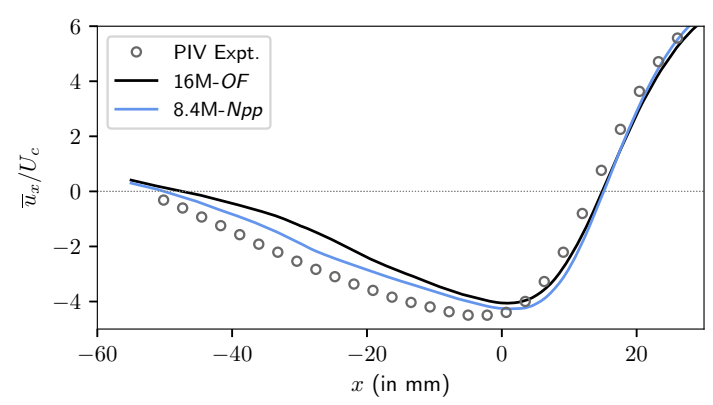

(a) Axial velocity along $r=5 \mathrm{~mm}$ line.

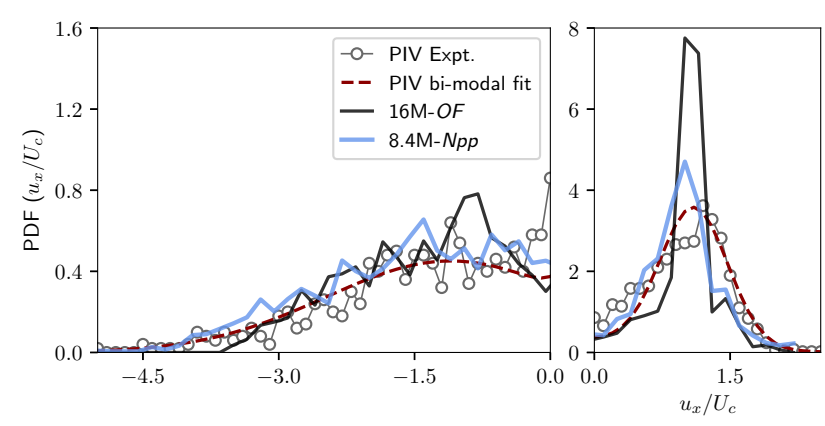

(b) PDF comparison at $x=-55 \mathrm{~mm}, r=0$.

Figure 5: Comparison of the second-order OpenFoam and P4 Nektar++ port-flow case results.

it was around 2. Finally, OF smoothly runs in this case with blended (10\% upwind and $90 \%$ central) scheme for the advection term. The time-step is $2.5 \times 10^{-6} \mathrm{~s}$ for both $O F$ and Npp.

An estimate of the flow-through time $t_{F}$ is $D_{s} / U_{s}$. With $D_{s}=37.63 \mathrm{~mm}$ and $U_{s}=1.99, t_{F}=0.019 \mathrm{~s}$. The flow is developed for $50 t_{F}$ and the statistics are collected for $150 t_{F}$. In addition, averaging in the azimuthal direction is applied. The cost incurred for collecting the statistics is around 30,000 core hours.

\section{RESULTS}

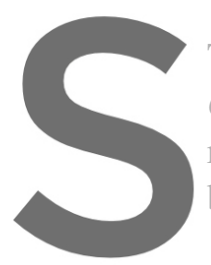

This section presents OpenFoam and fifth-ord radial velocity componen by the overbar $($ ).

4.1 Port flow
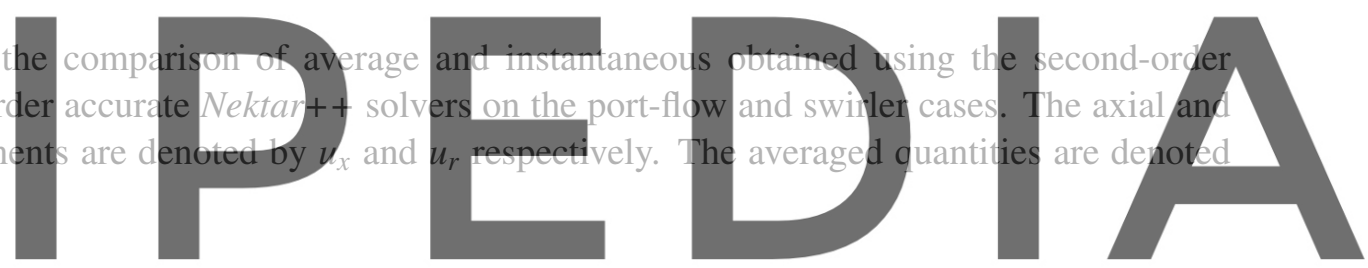

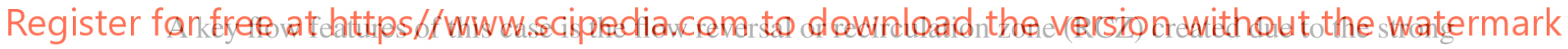

impingement of the jets [14]. The reference measurements [15] give the location of impingement point as $r=0, x=14 \mathrm{~mm}$. These features are illustrated in Figure 1. Both the simulations provide this location around $x=15 \mathrm{~mm}$. Further, the simulations under-predict the size of the reverse flow zone, $O F$ more than $N p p$. This is shown by plotting the mean axial velocity along a line parallel to central axis at $r=5 \mathrm{~mm}$ in Figure 5a. Recall that the location of the ports is $-10 \leq x \leq 10 \mathrm{~mm}$. Comparing the two simulations, in the RCZ region (where $\bar{u}_{x}$ is negative), it is seen that the Npp agrees more with the reference PIV. The reason may be understood from the following.

A distinguishing unsteady feature of the present flow configuration is slow $(O \approx 1 \mathrm{~Hz})$ periodic growth and shrinkage of the RCZ. The original study [14], used the probability density function (PDF) of $u_{x}$ at an upstream location to quantify this phenomenon. A bi-modal behaviour was identified $[14,16]$. The PDF obtained from the reference PIV [15], its bi-modal fit and the current LES data are plotted at $x=-55 \mathrm{~mm}, r=0$ in Figure $5 \mathrm{~b}$ (this location is pictured as 'Point probe' in Figure 1). The two parts of the plot address the two peaks; note the change in the ordinate-scale. Both the simulations reproduce the general shape of the PDF, however, there are stark differences. Starting from the left, note that $O F$ has not registered any events for $u_{x} / U_{c}<-3.6$, as opposed to the experiment and $N p p$. Thereafter, the shape 


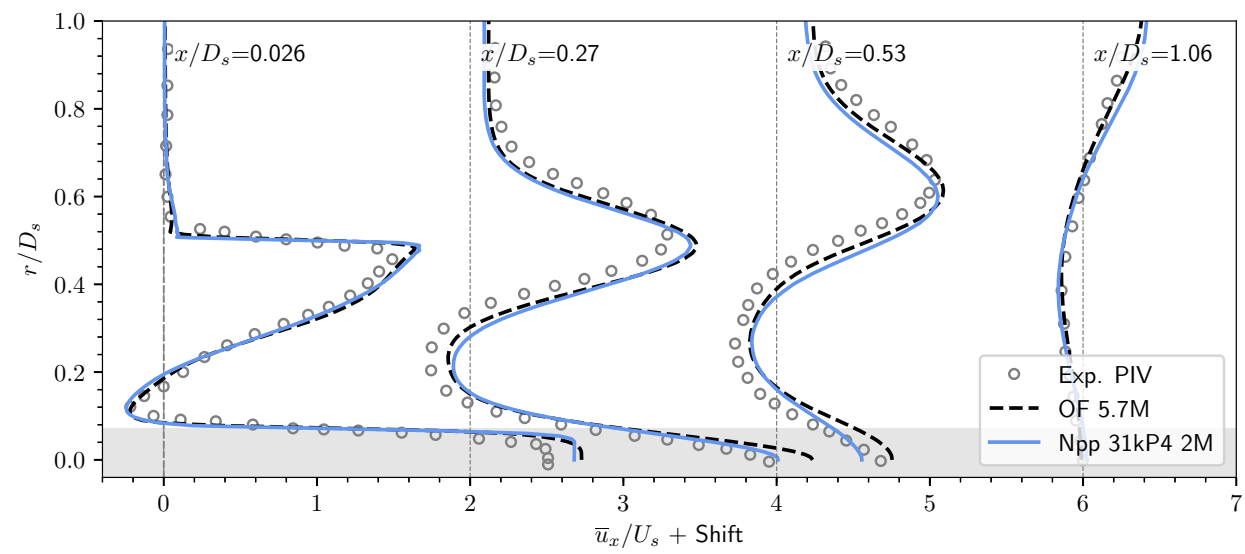

(a) Mean axial velocity along radial lines. 'Shift' refers to shifting of origin on the abscissa to vertical dashed lines. Grey shaded region represents the central jet.

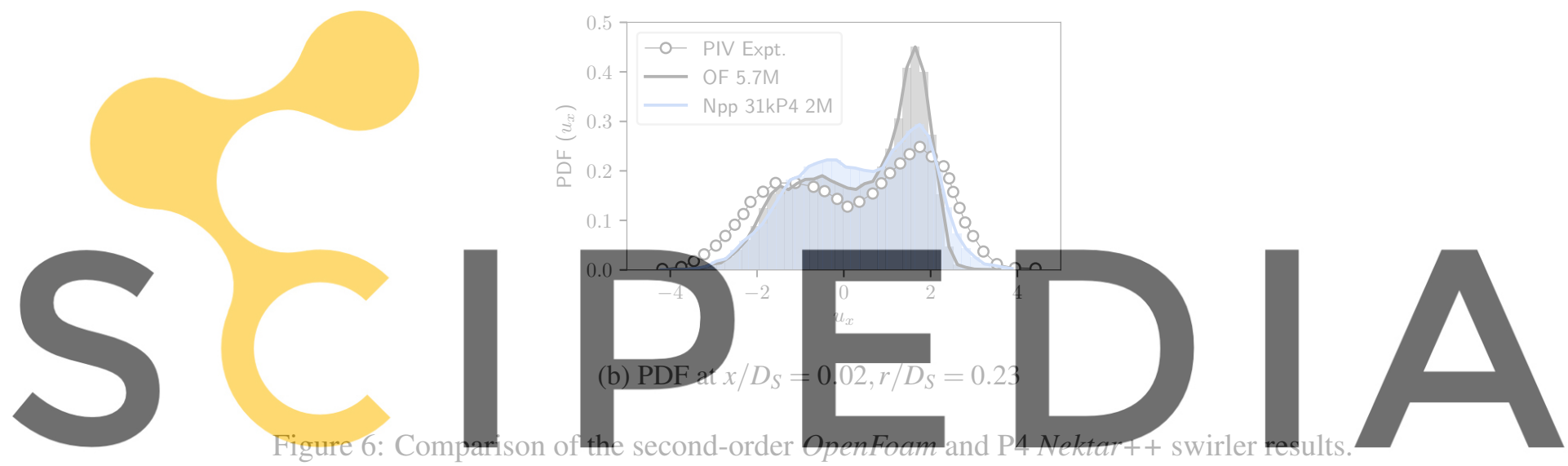

\section{Register for free at https//www scipedia.com, to download the version without the watermark \\ peak. On the right sub-plot, Npp clearly agrees better with the experiment than $O F$. Physically, it means}

that $O F$ predicts the direction of axial-velocity unduly positive, or in other words, the penetration of RCZ is underestimated. This corroborates the observation made in the $\bar{u}_{x}$ plot in Figure 5a. This behaviour may be attributed to the excessive dissipation of the turbulence kinetic energy that, if estimated correctly, would allow the fluctuations to 'drill deeper' in the upstream direction.

In summary, the high-order Npp simulation reproduced both the mean and unsteady flow physics of the RCZ more accurately. This outcome is significant, given the vital role that RCZ unsteadiness plays in gas turbine combustors [1].

\subsection{Swirler}

As indicated in Figure 3a, there exists a central recirculation (or reverse flow) zone that intrudes into the swirler passage. This has an impact on mixing and combustion charateristics and hence it is important to predict it correctly $[20,21]$. The reverse flow is represented by the mean axial velocity, $\bar{u}_{x}$, which is plotted along four radial lines (drawn in Figure 4) in Figure 6a. On the station just downstream 
the swirler passage $\left(x / D_{s}=0.02\right)$, the $\bar{u}_{x}$ is negative for a small distance and this is captured well by both the simulations. Small difference may be seen in the prediction of jet profile which persists in the downstream stations. This suggests that high-order Npp predicts the mixing of jet shear layer slightly better. Away from the axis, both the LES results are fairly close to reference experiment. This is the case for the radial and azimuthal velocity components as well (not included here).

Given the existence of large coherent PVC structures and their effect on mixing [20] and flow stability, flow data is collected over several relevant point locations. One such location is on the inner shear layer of the swirl stream, slightly downstream the swirler passage, i.e. $x / D_{s}=0.02, r / D_{s}=0.23$ as highlighted in Figure 4. The passing frequency of the PVCs is predicted equally well by the two LES simulations as compared to the experiment (omitted here). However, their effect on the instantaneous flow differs as shown by the PDF of the axial velocity in Figure 6b. The two peaks indicate that there are two dominating flow states at this location. These states have been attributed to the periodic presence and absence of the PVCs [20]. It is clear from Figure $6 \mathrm{~b}$ that $O F$ over-predicts the likelihood of positive $u_{x}$, which indicates that either the intensity or the region of influence of PVCs is not represented correctly. On the other hand, Npp is much closer to the experimental data. Further investigation is required to fully understand this behaviour.

In summary, both low- and high-order LES reproduced the mean velocity and PVC frequency with a similar accuracy. However, the local statistical flow structure, which is important for mixing and combustion, is reproduced significantly better by the high-order simulation.

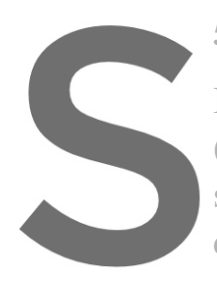

5 CONCLUSIONS

In order to clarify on the

(LES) for industrially re

second-order OpenFoan

directly relevant to gas tu
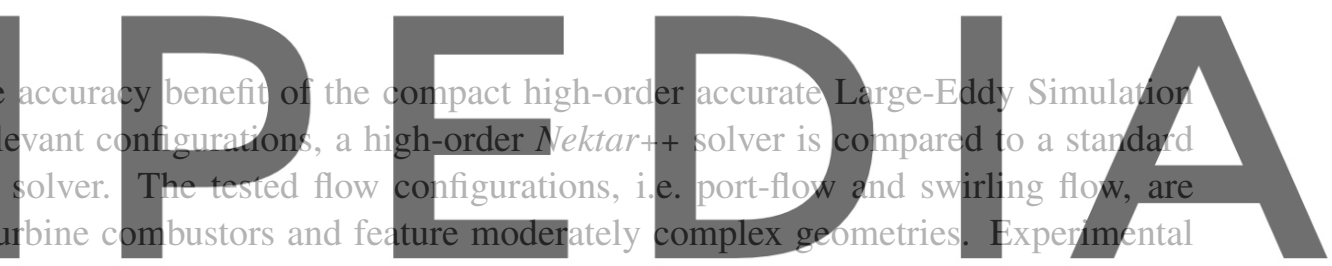

data from previous works is used as reference. The accuracy of the mean and instantaneous turbulence

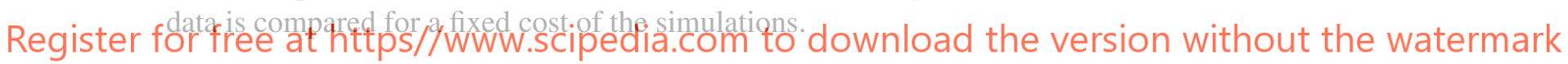

In the port-flow case, a key flow feature (reverse flow zone) is significantly more accurately reproduced

by the high-order simulation, both in terms of mean and unsteady velocity statistics. On the other hand, in the swirler case, the difference between the mean velocity results from the low- and high-order LES is minor. However, the unsteady effects of precessing vortices (an important feature) is shown to be more accurately resolved by the high-order simulation. This benefit may be larger on fully unstructured meshes in the swirler case.

The work has shown that for turbulent combustion applications, the benefit of the high-order approach lies in improving the accuracy of not only small-scale but also large-scale unsteady flow features, that could be critical in determining the combustion behaviour. In this light, finite-element type compact high-order methods are a credible means to obtain considerable accuracy benefits for practical turbulent combustion system applications.

\section{REFERENCES}

[1] J. J. McGuirk. The aerodynamic challenges of aeroengine gas-turbine combustion systems. Aeronautical Journal, 118:557-599, 2014. 
[2] Z. J. Wang, K. Fidkowski, R. Abgrall, et al. High-order CFD methods: current status and perspective. International Journal for Numerical Methods in Fluids, 72:811-845, 2013.

[3] F. Capuano, A. Palumbo, and L. de Luca. Comparative study of spectral-element and finite-volume solvers for direct numerical simulation of synthetic jets. Computers and Fluids, 179:228-237, 2019 .

[4] B. C. Vermeire, F. D. Witherden, and P. E. Vincent. On the utility of GPU accelerated high-order methods for unsteady flow simulations: A comparison with industry-standard tools. Journal of Computational Physics, 2017.

[5] V. Saini, H. Xia, and G. J. Page. Accuracy and Efficiency Comparison of Finite-Volume And Spectral/hp Methods for LES of a Combustor Relevant Geometry. International Conference on Computational Heat and Mass Transfer (ICCHMT), Rome, 2019.

[6] C. D. Cantwell, D. Moxey, A. Comerford, A. Bolis, et al. Nektar++: An open-source spectral/hp element framework. Computer Physics Communications, 192:205-219, 2015.

[7] H. G. Weller, G. Tabor, H. Jasak, and C. Fureby. A tensorial approach to computational continuum mechanics using object-oriented techniques. Computers in Physics, 12(6):620, 1998.

[8] T. Dairay, E. Lamballais, S. Laizet, and J. C. Vassilicos. Numerical dissipation vs. subgrid-scale modelling for large eddy simulation. Journal of Computational Physics, 337:252-274, 2017.

[9] F. Nicoud and F. Ducros. Subgrid-Scale Stress Modelling Based on the Square of the Velocity Gradient Tensor. Flow, Turbulence and Combustion, 62(3):183-200, 1999.

[10] G-S. Karamanos and G.E. Karniadakis. A Spectral Vanishing Viscosity Method for Large-Eddy Simulations. Journal of Computational Physics, 163(1):22-50, 2000.

[11] J. W. Lombard, D. Moxey, S. J. Sherwin, J. F. A. Hoessler, S. Dhandapani, and M. J. Taylor. Implicit Large-Eddy Simulation of a Wingtip Vortex. AIAA Journal, 54(2):506-518, 2016.

[12] T. Dairay, V. Fortuné, E. Lamballais, and L. E. Brizzi. LES of a turbulent jet impinging on a heated wall using high-order numerical schemes. International Journal of Heat and Fluid Flow, 50:177-187, 2014.

[13] R. C. Moura, S. J. Sherwin, and J. Peiró. Eigensolution analysis of spectral/hp continuous Galerkin approximations to advection-diffusion problems: Insights into spectral vanishing viscosity. Journal of Computational Physics, 307:401-422, 2016.

[14] A. Spencer and J.J. McGuirk. LDA Measurements of Feed Annulus Effects on Combustor Liner Port Flows. Journal of Fluids Engineering, 123:219-227, 2001.

[15] D. Hollis. Particle image velocimetry in gas turbine combustor flow fields. PhD thesis, Loughborough University, 2004.

[16] A. Spencer and V. Adumitroaie. Large Eddy Simulation of Impinging Jets in Crossflow. Proceedings of ASME Turbo Expo 2003, pages 1-8, 2003.

[17] D. J. Clayton and W. P. Jones. Large Eddy Simulation of Impinging Jets in a Confined Flow. Flow Turbulence Combustion, 77:127-146, 2006.

[18] A.K. Gupta, D.G. Lilley, and N. Syred. Swirl Flows. Energy and engineering science series. Abacus 
Press, 1984.

[19] Kristofer Midgley. An Isothermal Experimental Study of the Unsteady Fluid Mechanics of Gas Turbine Fuel Injector Flowfields. PhD thesis, Loughborough University, 2005.

[20] L. Cheng, M. Dianat, A. Spencer, and J. J. McGuirk. Validation of LES Predictions of Scalar Mixing in High-swirl Fuel Injector Flows. Flow, Turbulence and Combustion, 88:143-168, 2012.

[21] B. Janus, A. Dreizler, and J. Janicka. Experiments on swirl stabilized non-premixed natural gas flames in a model gasturbine combustor. Proceedings of the Combustion Institute, 31(2):30913098, 2007.

[22] K. Midgley, A. Spencer, and J. J. McGuirk. Unsteady Flow Structures in Radial Swirler Fed Fuel Injectors. Journal of Engineering for Gas Turbines and Power, 127(4):755-764, Oct 2005.

[23] A. Spencer, J. J. McGuirk, and K. Midgley. Vortex Breakdown in Swirling Fuel Injector Flows. Journal of Engineering for Gas Turbines and Power, 130(2):021503, 2008.

[24] D. Dunham, A. Spencer, J. J. McGuirk, and M. Dianat. Comparison of Unsteady Reynolds Averaged Navier-Stokes and Large Eddy Simulation Computational Fluid Dynamics Methodologies for Air Swirl Fuel Injectors. J. Eng. Gas Turbines Power, 131-1, 2008. 